\title{
The meteorological model RAMS at Crati Scrl
}

\author{
S. Federico ${ }^{1,2}$, E. Avolio ${ }^{1,3}$, C. Bellecci ${ }^{1,3}$, and M. Colacino ${ }^{2}$ \\ ${ }^{1}$ CRATI Scrl, c/o Università della Calabria, Rende (CS), Italy \\ ${ }^{2}$ CNR-ISAC, via del Fosso del Cavaliere 100, Rome, Italy \\ ${ }^{3}$ Facoltà di Ingegneria-Università di "Tor Vergata", via del Politecnico 1, Rome , Italy
}

Received: 24 October 2004 - Revised: 11 March 2005 - Accepted: 14 March 2005 - Published: 30 May 2005

\begin{abstract}
At Crati Scrl an operational version of RAMS 4.3 (Regional Atmospheric Modeling System) was implemented in January 2001. This paper aims to give a first assessment of model performances for quantitative precipitation forecast (QPF). In essence, the effects of enhanced horizontal grid resolution over Calabria, using a $6 \mathrm{~km}$ spacing domain nested in a $30 \mathrm{~km}$ resolution parent grid, is studied.

To cope with this problem two integrations sets are discussed using two model resolutions. Integrations are performed daily for six months. ECMWF 12:00 UTC forecast cycle is used for initial and dynamic boundary conditions.

Performances are evaluated by scores computed from model outputs and raingauge measurements coming from Calabrian regional network.
\end{abstract}

\section{Introduction}

The aim of this paper is to give a first assessment of RAMS accuracy for quantitative precipitation forecast (QPF) over Calabria, the southernmost tip of Italian peninsula. Improving QPF is one of the most desired aspect in numerical weather prediction. Precipitation is a difficult field to predict quantitatively for several reasons. First of all the process depends on several factors as temperature, humidity, winds in highly nonlinear ways. Another complication is introduced by variable physiography, i.e. surface features such as topography, land water boundaries, vegetation and soil parameters. All those features are important for Calabria, Fig. 1, and the presence of mountains may act to enhance rainfall by several mechanisms including low-level convergence associated with flow deflection around the topography (Buzzi et al., 1998; Rotunno and Ferretti, 2001; Smith, 1979) and terrain-triggered convection (Federico et al., 2003).

Among others, model resolution over complex terrain is claimed as an important factor to better represent small scale

Correspondence to: S. Federico

(s.federico@crati.it) variations not considered in global scale models (Gaudet and Cotton, 1998). This is the subject of this paper. To investigate it, we use RAMS model in two different configurations for six months, form 1 November 2000 to 30 April 2001. Domains are shown in Fig. 2. First configuration uses the first domain only, i.e. grid 1, while second set up uses both domains, i.e. grid 1 and grid 2, via two way nesting. Horizontal resolutions are $30 \mathrm{~km}$ and $6 \mathrm{~km}$ for first and second grid, respectively. Hereafter we will refer to these set ups as CC (Coarse Configuration) and FC (Fine Configuration).

Forecasts are compared over the grid 2 domain by scores evaluated from model outputs and raingauge measurements available from ARPACAL (Calabrian regional agency for environmental protection) network. Data are daily cumulated rainfall. Scores difference is assessed by the application of the statistical test proposed by Hamill (1999).

\section{Model set-up}

The following is a brief description of model set up including options selected. For details on RAMS the reader should refer to Cotton et al. (2003).

Horizontal grids have been introduced in the previous section. Twenty five vertical levels, up to $12500 \mathrm{~m}$ in the terrain following coordinate system, are used. Levels are not equally spaced: within the PBL (Planetary Boundary Layer) layers run about 50-200 $\mathrm{m}$ tick, while in the middle and upper troposphere they are $1000 \mathrm{~m}$ tick.

The parameterization of surface-atmosphere diabatic processes is described in Walko et al. (2000). Non convective precipitation is computed from explicit prognostic equations for eight hydrometeors: total water, rain, pristine ice, cloud particles, ice, snow, hail and aggregates. A generalized gamma function is used to describe hydrometeors size distribution. Convective precipitation is parameterized following Molinari and Corsetti (1985) who proposed a simplified form of the Kuo scheme that accounts for updrafts and downdrafts. 


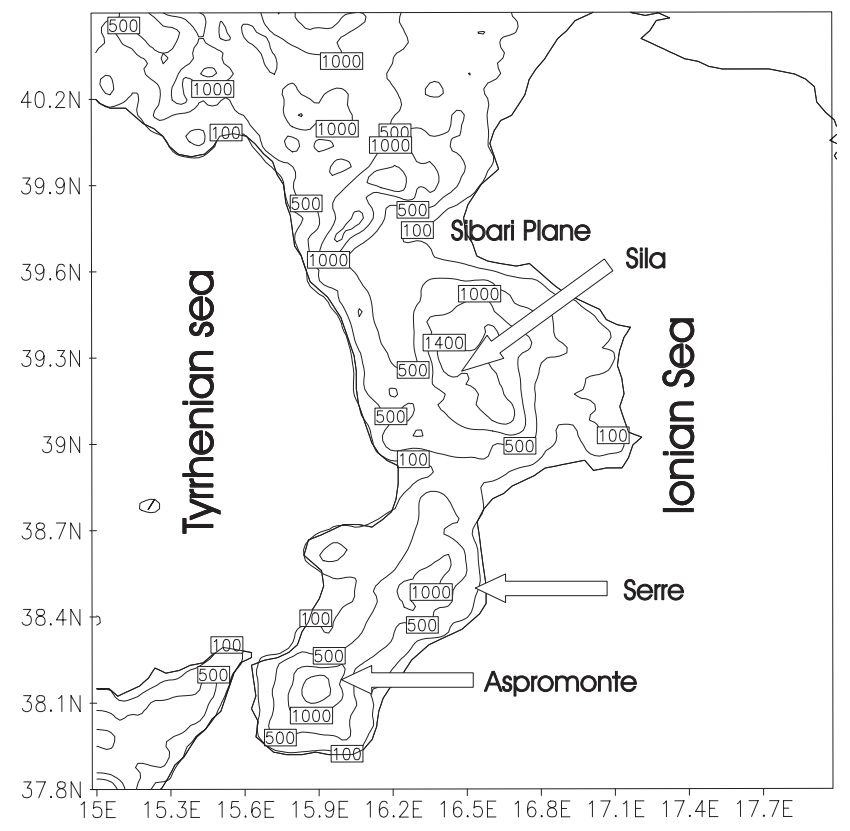

Fig. 1. Topography of Calabria averaged over $10 \mathrm{~km}^{2}$. Main features are also reported. Contours: $100 \mathrm{~m}, 500 \mathrm{~m}, 1000 \mathrm{~m}$ and $1400 \mathrm{~m}$

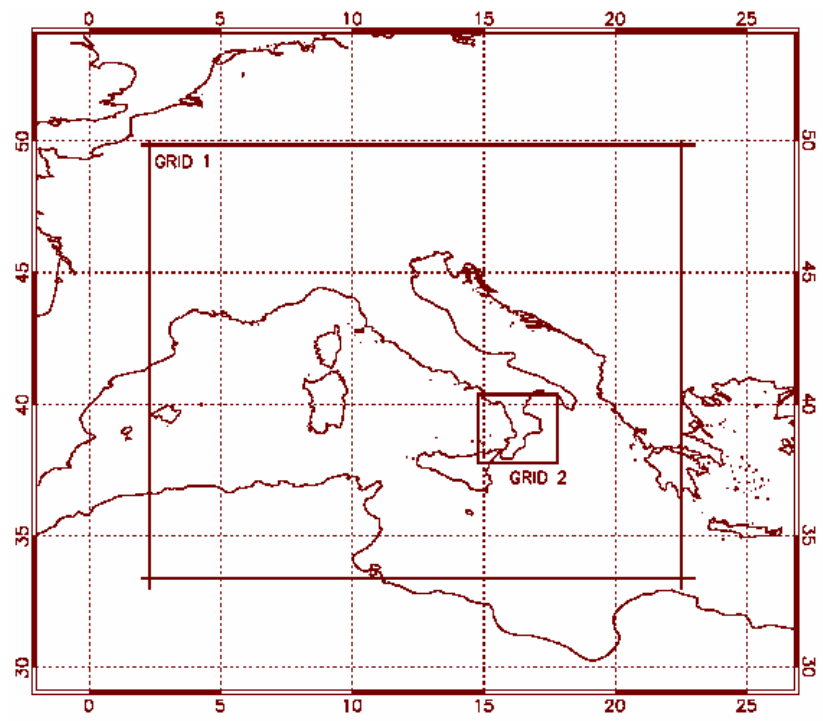

Fig. 2. Model domains. First grid is used by CC and FC forecasts and has $30 \mathrm{~km}$ horizontal spacing. Second grid is used by FC forecast only and has $6 \mathrm{~km}$ horizontal spacing.

Initial and dynamic boundary conditions are provided by 12:00 UTC ECMWF forecast cycle. Simulations duration is $60 \mathrm{~h}$. First $12 \mathrm{~h}$ are spin-up time so $48 \mathrm{~h}$ are available for discussion. This 2 day forecast is divided in two $24 \mathrm{~h}$ frames, the $1 \mathrm{D}$ and $2 \mathrm{D}$ forecasts.

To verify forecasts we use 36 raingauges dislocated, roughly uniformly, over Calabria. Each observation is assigned to the nearest grid point. If more raingauges are assigned to a grid point, the observed precipitation is their mean value.

\section{Methodology and data}

Scores are useful for evaluating deterministic gridded precipitation forecasts as those reported in this paper. They are generated as follows. Precipitation space is divided in four, mutually exclusive and exhaustive sets (contingency table):

1. hits $(a)$ is the number of grid points with both forecast and measurement greater than or equal to a threshold;

2. false alarms $(b)$ is the number of grid points with model above or equal to a threshold and measurement below;

3. misses (c) is the number of grid points with measurement above or equal to a threshold and forecast below;

4. correct no forecasts $(d)$ is the number of grid points with model and measurement below a threshold.

Every day of the verification period a contingency table is computed. Starting from contingency table we define the $B I A$ :

$B I A=\frac{a+b}{a+c}$

$B I A$ measures if the model overforecast or underforecast precipitation over an area. If $B I A>1$ the model overestimates the precipitation area, if $B I A<1$ the model underestimates this area. For a perfect forecast $B I A=1$. Another score used in this paper is the Equitable Threat Score $(E T S)$ :

$E T S=\frac{a-a_{r}}{a+b+c-a_{r}}$

where $a_{r}$ is the expected number of correct forecasts above the threshold in a random forecast, where forecast occurrence/non occurrence is independent from observation/non observation.

For a perfect forecast $E T S$ is equal to 1, while it is less than or equal to zero for a useless forecast.

To compare forecasts we use the hypothesis test developed by Hamill (1999) that is based on resampling. The methodology is also applied in Accadia et al. (2003) and will not be described in this paper. The null hypothesis of the resampling test is that scores differences of competitor forecasts, computed from a sum of daily contingency table samples over all the case days, are zero. Resampled test statistics consistent with the null hypothesis are generated after randomly choosing either one or the other model on each day and summing contingency table elements, i.e. vectors of contingengy tables elements $(a, b, c, d)$ of competitor forecasts are randomly shuffled on each day and statistics (BI A,ETS) recomputed from their sum. In this way we build the statistics PDFs that are used to accept or discard the null hypothesis at the test level (5\% in this paper). For a complete discussion of the methodology the reader is referred to Hamill (1999). 


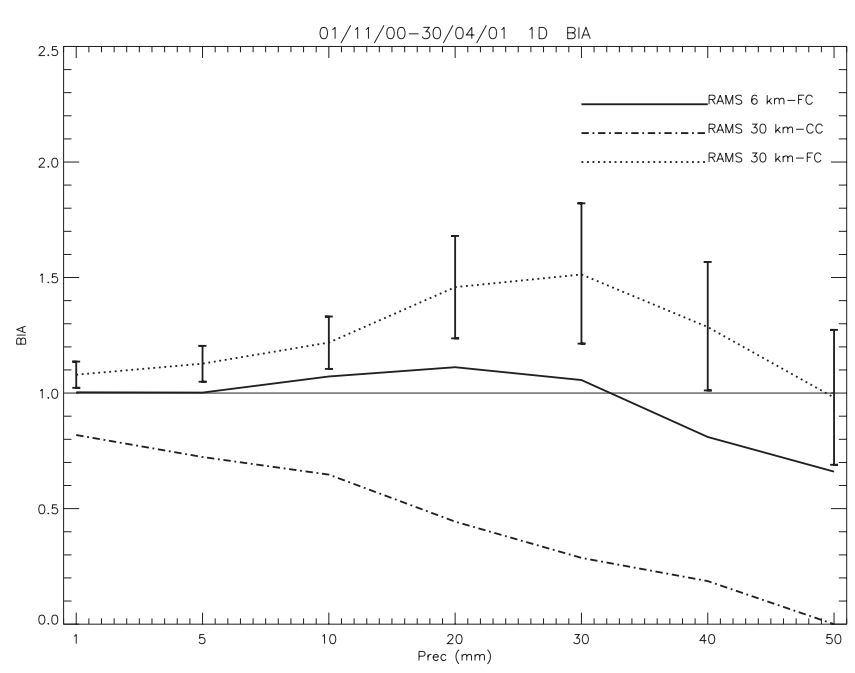

Fig. 3a. B I A for the first forecast day. Solid line is for FC second grid, dot-dashed line is for CC forecast, dotted line is for FC first grid. Error bars are computed according to Hamill test (1999).

\section{Results}

We study how BIA and ETS depends on several thresholds in a day: $1 \mathrm{~mm}, 5 \mathrm{~mm}, 10 \mathrm{~mm}, 20 \mathrm{~mm}, 30 \mathrm{~mm}, 40 \mathrm{~mm}$, $50 \mathrm{~mm}$. These values are a compromise between precipitation falling in a day over Calabria and the field variability.

Results for $B I A$ and $E T S$ are presented for the first day in Figs. 3a-b. Each figure has three curves. Dot-dashed line is the score for $\mathrm{CC}$ configuration, i.e. the grid 1 domain without the grid 2 included, dotted line is the score for FC first grid, i.e. when the grid 2 area is included via 2-way nesting, solid line is the score for the second FC grid. All the results are compared over the grid 2 domain. Error bars, reported for dotted line, are determined by the statistical test involving first FC grid and CC grid. Grid lattices are identical and comparison is straightforward. Indeed, with station reports simply box averaged, analysis smoothness will depend on the volume of data available per verification box. We compare first FC grid with CC because the data volume is the same for both grids.

Figure 3a shows that $B I A$ for CC configuration is, for every threshold, less than $B I A$ for both FC grids. Differences are statistically significant at $5 \%$ level for all thresholds. The use of a nested grid in FC forecast enhances precipitation on the first one by the two way interactive procedure (Walko et al., 1994). This produces a $B I A$ overestimation for the first FC grid. For thresholds larger than $20 \mathrm{~mm}, B I A$ low values for CC configuration are due to the poor resolution of this forecast that underestimates mountain heights and orographic uplift. For $50 \mathrm{~mm}$ threshold $B I A$ is zero for CC forecast. For lower thresholds $(\leq 20 \mathrm{~mm})$ rainfall triggering mechanisms are fundamental. A closer inspection of model outputs reveals that light precipitation events are sometimes missed by CC just because local physiographic features are not well represented in the model. These events are, at least

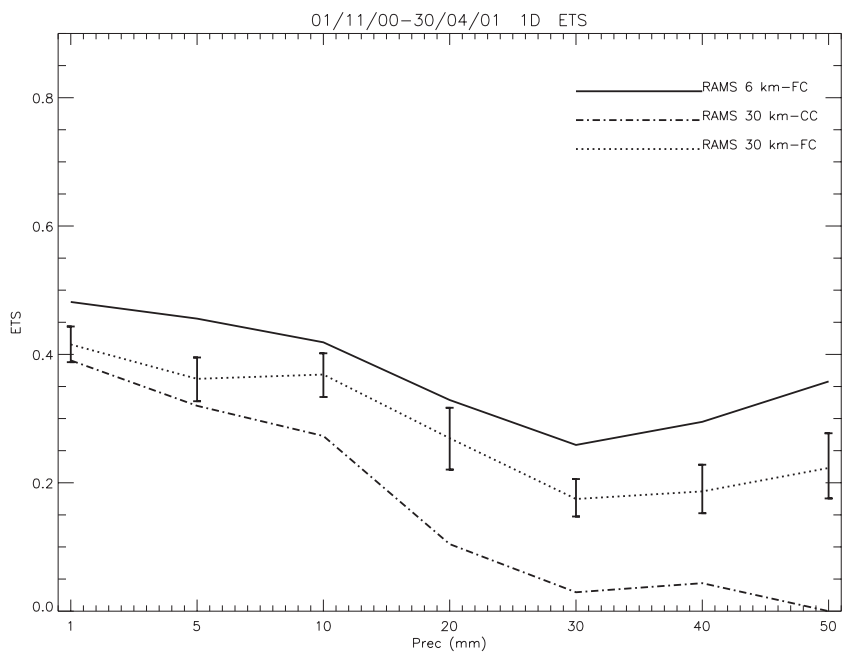

Fig. 3b. As in Fig. 3a for ETS.

partially, represented by FC (i.e. FC simulations hits are not zero). As large scale forcing is the same for both FC and CC forecasts, it follows that local orographic features are able to trigger precipitation for $\mathrm{FC}$ forecast due to the enhanced model resolution. So, BIA is underestimated by $\mathrm{CC}$ forecast at lower thresholds, whereas it is slightly overestimated by FC. Results for BI A show that local orography is fundamental for all rain amounts analyzed in this paper. This should be considered in weather related activities that do not necessarily involve large precipitation amounts.

ETS score is shown in Fig. 3b. FC forecast performs better for all thresholds. Differences are statistically significant, if we exclude $1 \mathrm{~mm}$ rainfall. ETS score is zero for $50 \mathrm{~mm} /$ day threshold and CC forecast is not useful for thresholds greater than $20 \mathrm{~mm}$. FC first grid forecast has larger ETS values than CC but lower than its nested grid. However, it is useful for all thresholds, being well above the zero value. From Fig. $3 b$ it follows the ability of nested grid to better reproduce precipitation spots, as a consequence of the finer representation of Calabria physiographic features.

Figure 4 shows scores for 2D forecast. There is a general increase of BIA compared to the first forecast day. CC and FC set ups are different, at 5\% level, and FC performs better.

$E T S$, Fig. 4b, reinforces results for first forecast day and FC performs better for all thresholds, even if differences are not statistically significant for $1 \mathrm{~mm}$ and $5 \mathrm{~mm}$. Coarse configuration ETS is close to zero for $50 \mathrm{~mm}$ threshold and shows that this model is useless at high precipitation amounts, at least for the integration period considered in this paper.

\section{Conclusions}

In this paper we investigated performances of RAMS model for quantitative precipitation forecast over Calabria. Two different model configurations were tested in order to assess the impact of enhanced horizontal grid resolution on QPF. 


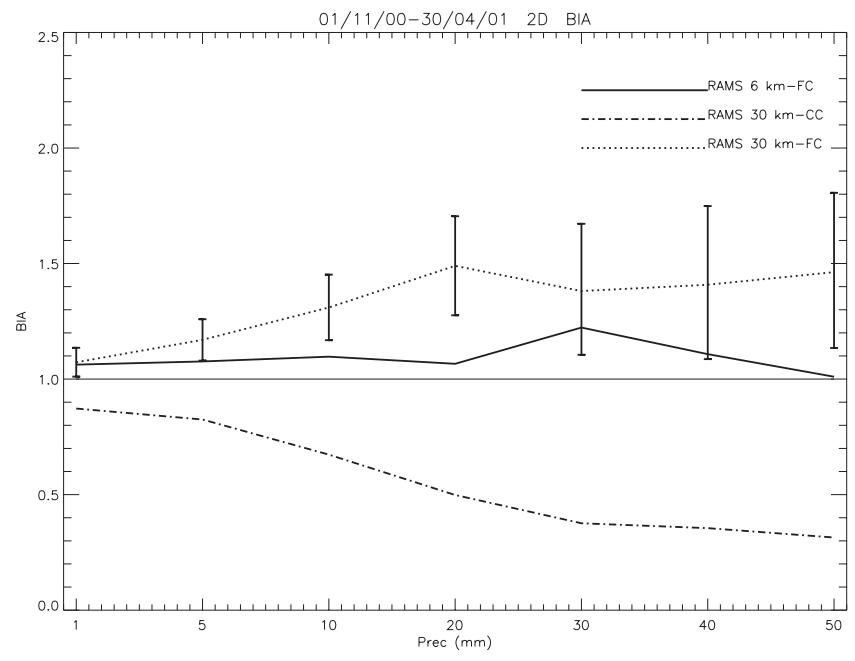

Fig. 4a. As in Fig. 3a for second integration day.

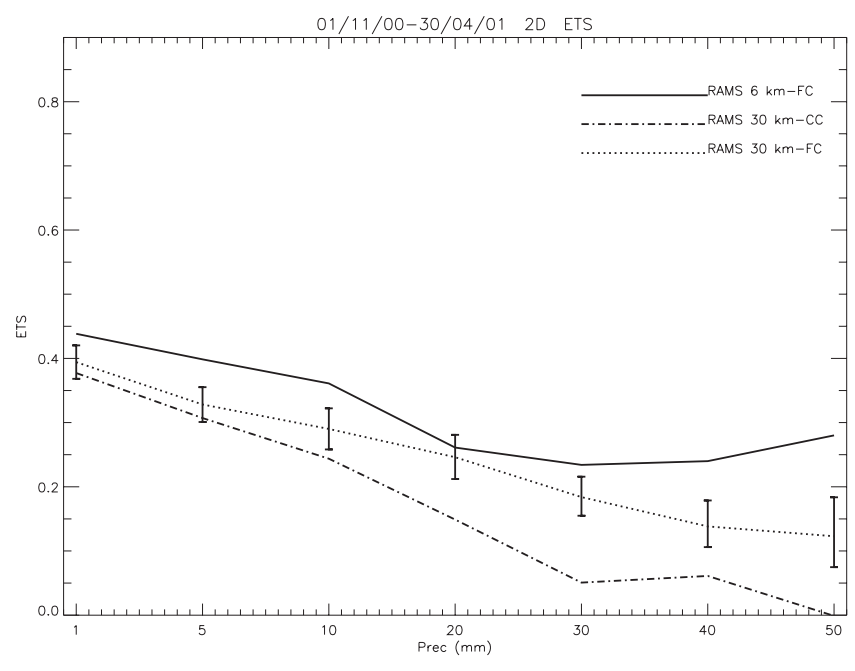

Fig. 4b. As in Fig. 3b for second integration day.

The use of a nested grid over Calabria is useful for all precipitation thresholds considered in this paper. Resolution enhancement produces a better representation of physiographic features and results in better performances not only for large precipitation amounts but also for light rain. Better performances of fine resolution forecast are reported for both integration days and performances are statistically significant at $5 \%$ rejection level.

Acknowledgements. This work was realized in the framework of the project "Sviluppo di Distretti Industriali per le Osservazioni della Terra" funded by "Ministero dell'Università e della Ricera Scientifica". We are grateful to ARPACAL for surface data. We are grateful to the Italian Air Force and ECMWF for the access to the MARS database.

Edited by: L. Ferraris

Reviewed by: anonymous referees

\section{References}

Accadia, C., Casaioli, M., Mariani, S., Lavagnini, A., Speranza, A., De Venere, A., Inghilesi, R. , Ferretti, R., Paolucci, T., Cesari, D., Patrono, P., Boni, G., Bovo, S., and Cremonini, R.: Application of a statistical methodology for limited area model intercomparison using a bootstrap technique, Nuov. Cim. C , 26 C, 61-77, 2003.

Buzzi, A., Tartaglione, N., and Malguzzi, P.: Numerical Simulations of the 1994 Piedimont flood: role of Orography and moist precesses, Mon. Wea. Rev., 126, 2369-2383, 1998.

Cotton, W. R., Pielke, R. A. Sr., Walko, R. L., Liston, G. E., Tremback, C. J., Jiang, H., McAnelly, R. L., Harrington, J. Y., Nicholls, M. E., Carrio, G. G., and McFadden, J. P. : RAMS 2001: Current satus and future directions, Meteor. Atm., 82, 529, 2003.

Federico, S., Bellecci, C., and Colacino, M.: Quantitative precipitation of the Soverato flood: the role of orography and surface fluxes. Nuov. Cim. C, 26 C, 7-22, 2003.

Gaudet, B. and Cotton, W. R.: Statistical Characteristics of a realtime precipitation forecasting model, Weather For., 13, 966982, 1998.

Hamill, T. M.: Hypothesis tests for evaluating numerical precipitation forecasts, Weather For., 14, 155-167, 1999.

Molinari, J. and Corsetti, T.: Incorporation of cloud-scale and mesoscale down-drafts into a cumulus parametrization: results of one and three-dimensional integrations, Mon. Wea. Rev., 113, 485-501, 1985.

Rotunno, R. and Ferretti, R.: Mechanism of intense Alpine rainfall, J. Atmos. Sci., 58, 1732-1750, 2001

Smith, R. B.: The influence of mountains on the atmosphere, Adv. Geophys., 21, Academic Press, 87-230, 1979.

Walko, R. L., Tremback, C. J., Pielke, R. A., and Cotton, W. R.: An interactive nesting algorithm for stretched and variable nesting ratio, J. Appl. Met., 34, 994-999, 1994.

Walko, R. L., Band, L. E., Baron, J., Kittel, T. G., Lammers, R., Lee, T. J., Ojima, D., Pielke, R. A. Sr., Taylor, C., Tague, C., Tremback, C. J., and Vidale, P. L.: Coupled Atmosphere-BiosphereHydrology Models for environmental prediction, J. Appl. Met., 39, 931-944, 2000. 